\title{
NARD: whole-genome reference panel of 1779 Northeast Asians improves imputation accuracy of rare and low-frequency variants
}

\author{
Seong-Keun $\mathrm{YoO}^{1,2 \dagger}{ }^{\dagger}$, Chang-Uk Kim ${ }^{2,3 \dagger}$, Hie Lim Kim ${ }^{4,5 \dagger}$, Sungjae Kim ${ }^{2,3 \dagger}{ }^{\dagger}$, Jong-Yeon Shin ${ }^{2}$, Namcheol Kim², \\ Joshua Sung Woo Yang ${ }^{2}$, Kwok-Wai Lo ${ }^{6}$, Belong Cho ${ }^{7}$, Fumihiko Matsuda ${ }^{8}$, Stephan C. Schuster ${ }^{5,9}$, \\ Changhoon Kim², Jong-II Kim ${ }^{3,10}$ and Jeong-Sun Seo ${ }^{1,2,3,10,11^{*}}$ (D)
}

\begin{abstract}
Here, we present the Northeast Asian Reference Database (NARD), including whole-genome sequencing data of 1779 individuals from Korea, Mongolia, Japan, China, and Hong Kong. NARD provides the genetic diversity of Korean $(n=850)$ and Mongolian $(n=384)$ ancestries that were not present in the 1000 Genomes Project Phase 3 (1KGP3). We combined and re-phased the genotypes from NARD and 1KGP3 to construct a union set of haplotypes. This approach established a robust imputation reference panel for Northeast Asians, which yields the greatest imputation accuracy of rare and low-frequency variants compared with the existing panels. NARD imputation panel is available at https://nard.macrogen.com/.
\end{abstract}

Keywords: Whole-genome sequencing, Reference panel, Genotype imputation, Northeast Asians, East Asians

\section{Background}

During the past decade, the reference panels with population-scale whole-genome sequencing (WGS) have enabled the extensive human genetic research $[1,2]$. They have played an imperative role in the genetic research, especially for genotype imputation in genome-wide association studies (GWAS). The most commonly used imputation panels were constructed by the 1000 Genomes Project Phase 3 (1KGP3) and Haplotype Reference Consortium (HRC) studies, which are publicly available for researchers. As genotype imputation is an essential step to increase the power of GWAS in a cost-efficient way, the confidence of imputed genotypes is the most important. To improve the quality of imputation in genetic studies, the large-scale population-specific reference panels with deep sequencing coverage are required. Accordingly, several research groups have generated large-scale WGS data to build their own population-specific reference panels [3-10].

\footnotetext{
* Correspondence: jeongsunseo@gmail.com

†'Seong-Keun Yoo, Chang-Uk Kim, Hie Lim Kim and Sungjae Kim contributed equally to this work.

${ }^{1}$ Precision Medicine Center, Seoul National University Bundang Hospital, 172 Dolma-ro, Seongnam, Bundang-gu, Gyeonggi-do 13605, Republic of Korea ${ }^{2}$ Precision Medicine Institute, Macrogen Inc., Seongnam, Republic of Korea Full list of author information is available at the end of the article
}

Despite Northeast Asians account for $21.5 \%$ of worldwide population (http://www.worldometers.info/world-population), the majority of genetic studies and reference panels are biased to European ancestries [11]. There are some population-scale studies for building reference panels of Han Chinese (CHN), Japanese (JPN), Mongolians (MNG), and Koreans (KOR), but several issues, including public unavailability $[6,10,12,13]$, inadequate sequencing coverage $[12,14]$, small sample size $[10,15]$, and restriction to exonic regions $[16,17]$, need to be resolved for the solid imputation reference panel. Therefore, constructing a large-scale whole-genome reference panel covering the diverse population groups in Northeast Asia with deep sequencing coverage is still necessary to allow dense and accurate genotype imputation for the genetic research in these populations.

Here, we describe the Northeast Asian Reference Database (NARD), consisting of 1779 individuals from Korea, Japan, Mongolia, China, and Hong Kong. The goal of this study is to establish a high-quality population-specific reference panel for the genetic studies and precision medicine in Northeast Asia without the aforementioned issues.

(c) The Author(s). 2019 Open Access This article is distributed under the terms of the Creative Commons Attribution 4.0 International License (http://creativecommons.org/licenses/by/4.0/), which permits unrestricted use, distribution, and reproduction in any medium, provided you give appropriate credit to the original author(s) and the source, provide a link to the Creative Commons license, and indicate if changes were made. The Creative Commons Public Domain Dedication waiver (http://creativecommons.org/publicdomain/zero/1.0/) applies to the data made available in this article, unless otherwise stated. 


\section{Construction and content Variant statistics}

The NARD contains 1779 Northeast Asians including KOR $(n=850)$, JPN $(n=396)$, MNG $(n=384)$, CHN $(n=91)$, and Hong Kongese (HKG, $n=58)$ with deep $(20 \times \leq, n=834)$ or intermediate $(10 \times \sim 20 \times, n=945)$ sequencing coverages (Additional file 1: Figure S1, Additional file 2: Table S1). Initially, WGS was performed on 1781 Northeast Asians, but two MNG samples with low variant count and an abnormal ratio of heterozygous to homozygous genotypes (Het/Hom) were discarded in the subsequent analysis (Additional file 1: Figure S2). We evaluated potential bias from inconsistent sequencing coverage of samples and found no significant correlation (Pearson correlation coefficient) between the sequencing depth and the number of variants: single nucleotide polymorphism (SNP; $R=0.15$ ) and short insertion/deletion (indel; $R=-0.20$ ). Also, transition to transversion $(\mathrm{Ti} / \mathrm{Tv})$ ratios were consistent across the samples (2.1 on average; Additional file 1: Figure S3). The Het/Hom ratios (1.4 on average; Additional file 1: Figure S4) and the number of loss-of-function variants (35.4 on average; Additional file 1: Figure S5) in the NARD were similar to those in East Asians from the 1KGP3 (1.3 and 36.9 on average for each). Also, 99.2\% of the variants passed Hardy-Weinberg equilibrium (HWE) test $\left(P>1 \times 10^{-5}\right.$; Additional file 1: Figure S6).

In the NARD, a total of 40.6 million SNPs and 3.8 million indels were discovered, and $77.1 \%$ were singletons or rare variants (minor allele frequency $[\mathrm{MAF}]<0.5 \%$; Table 1). On average, 3.3 million SNPs and 0.3 million indels were found for each individual. We identified 15.4 million novel SNPs $(37.8 \%$ of the total) in the NARD (Additional file 1: Figure S7a). Among them, $45.0 \%$ were specific to KOR, likely due to their large sample size, and $12.6 \%$ were found across populations (Additional file 1: Figure S7b). The majority of novel SNPs were singletons or rare variants and located in noncoding regions (Additional file 1: Figure S7c). We found the high integrity of our WGS variant call pipeline; the genotype concordance between WGS and Illumina Omni $2.5 \mathrm{M}$ array of $86 \mathrm{CHN}$ samples from the NARD was $99.6 \%$ (Additional file 2: Table S2).

\section{Ancestry composition of NARD}

We examined the ancestry composition of individuals in the NARD to illustrate how it covers the genetic diversity that was not present in other reference panels. From the principal component analysis (PCA) result of global human populations, individuals from the NARD were closely related to East Asians from the 1KGP3 as expected (Fig. 1a). MNG were separately clustered and positioned between East Asian and non-African populations as previously reported [10]. When we applied PCA to only Northeast and Southeast Asians, a clear population differentiation pattern was observed among them (Fig. 1b); MNG were most distinct from other populations based on PC1, and PC2 separated KOR, JPN, and mainland East Asians including Chinese Dai in Xishuangbanna (CDX), Han Chinese in Beijing (CHB), Han Chinese in Shanghai (CHS), HKG, and Kinh in Ho Chi Minh City (KHV). Interestingly, there were no overlapped samples between KOR and JPN except for a few outliers. This result implies that their ancestral compositions are distinctive enough to form separate clusters. Additionally, unsupervised ADMIXTURE analysis [18] supported the different ancestral components for each of KOR, MNG, JPN, and mainland East Asians (Fig. 1c). In the case of MNG, there were Buryats (BUR, $n=299$ ), Khalkha Mongols (KHA, $n=73$ ), and other Mongolians including Barga, Daringanga, Kazakh, Khoton, Uuld, Durvud, Khotogoid,

Table 1 Total number of variants in 1779 individuals by MAF and functional category

\begin{tabular}{|c|c|c|c|c|c|c|c|c|c|c|c|}
\hline \multirow[t]{3}{*}{ Type } & \multirow[t]{3}{*}{ Frequency ${ }^{a}$} & \multirow{3}{*}{$\begin{array}{l}\text { Number } \\
\text { of variants }\end{array}$} & \multicolumn{9}{|c|}{ Functional variation } \\
\hline & & & \multicolumn{4}{|c|}{ Protein coding region } & \multicolumn{5}{|c|}{ Non-coding region } \\
\hline & & & $\begin{array}{l}\text { Silent/ } \\
\text { nonframeshift }\end{array}$ & $\begin{array}{l}\text { Missense/ } \\
\text { frameshift }\end{array}$ & $\begin{array}{l}\text { Stoploss/ } \\
\text { Stopgain }\end{array}$ & Unknown & Intronic & Intergenic & Splicing & UTR & ncRNA \\
\hline \multirow[t]{5}{*}{ SNP } & Singleton & $17,811,366$ & 86,804 & 146,480 & 3722 & 2690 & $6,842,300$ & $9,370,754$ & 2110 & 247,422 & $1,109,084$ \\
\hline & Rare & $13,673,626$ & 54,642 & 87,791 & 1658 & 1917 & $5,270,353$ & $7,248,270$ & 1363 & 164,492 & 843,140 \\
\hline & Low & $3,430,315$ & 12,753 & 15,710 & 232 & 428 & $1,299,727$ & $1,851,373$ & 245 & 38,673 & 211,174 \\
\hline & Common & $5,727,339$ & 17,886 & 15,981 & 151 & 729 & $2,049,372$ & $3,228,994$ & 159 & 53,221 & 360,846 \\
\hline & Total & $40,642,646$ & 172,085 & 265,962 & 5763 & 5764 & $15,461,752$ & $21,699,391$ & 3877 & 503,808 & $2,524,244$ \\
\hline \multirow[t]{5}{*}{ Indel } & Singleton & $1,402,707$ & 3191 & 5068 & 157 & 129 & 558,772 & 717,182 & 517 & 27,748 & 89,943 \\
\hline & Rare & $1,376,996$ & 2733 & 2884 & 127 & 127 & 544,183 & 717,045 & 217 & 22,047 & 87,633 \\
\hline & Low & 452,337 & 634 & 827 & 37 & 37 & 173,946 & 241,506 & 61 & 6444 & 28,845 \\
\hline & Common & 569,436 & 422 & 369 & 18 & 89 & 207,132 & 317,135 & 145 & 7157 & 36,969 \\
\hline & Total & $3,801,476$ & 6980 & 9148 & 339 & 382 & $1,484,033$ & $1,992,868$ & 940 & 63,396 & 243,390 \\
\hline
\end{tabular}

${ }^{\mathrm{a}}$ Rare, $\mathrm{MAF}<0.5 \%$; low, $0.5 \% \leq \mathrm{MAF}<5 \%$; common, MAF $\geq 5 \%$ 
a

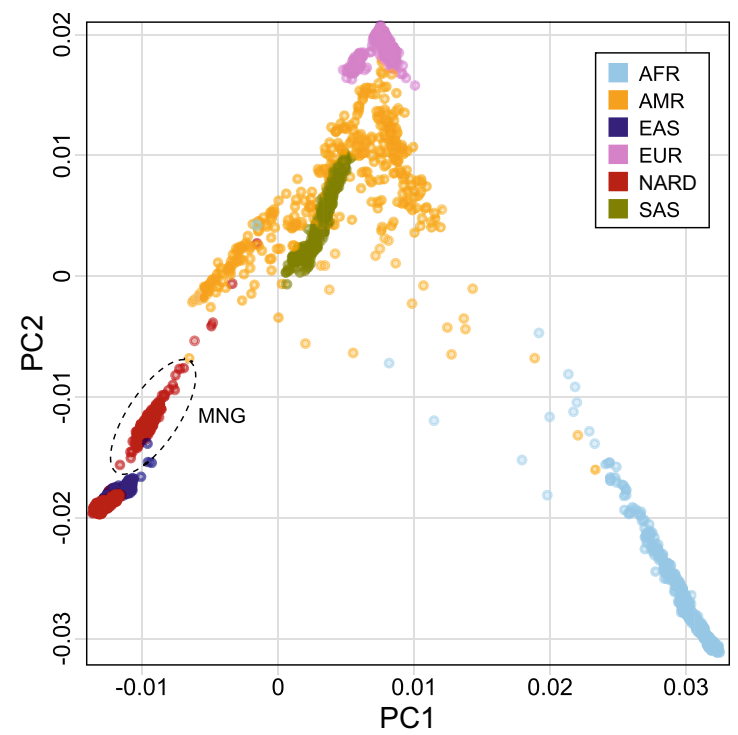

b

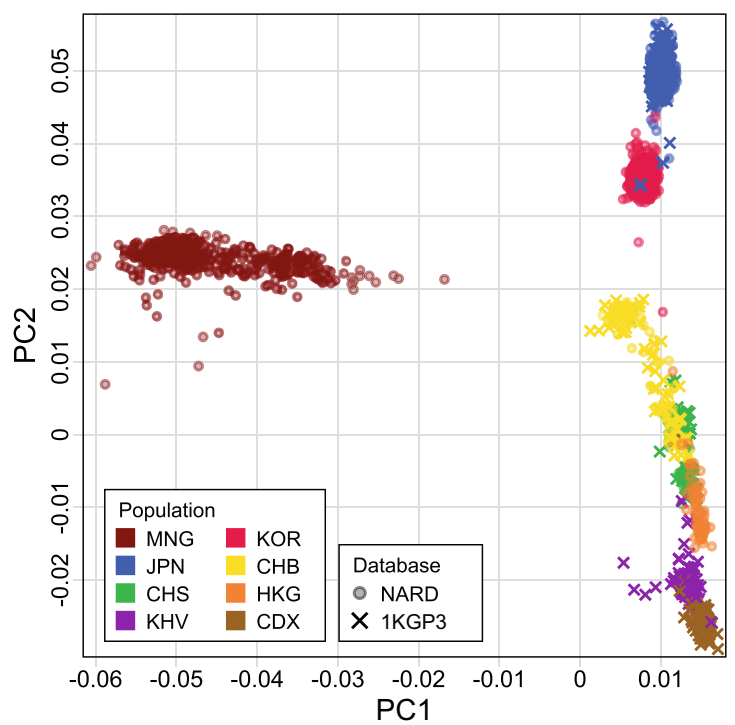

C

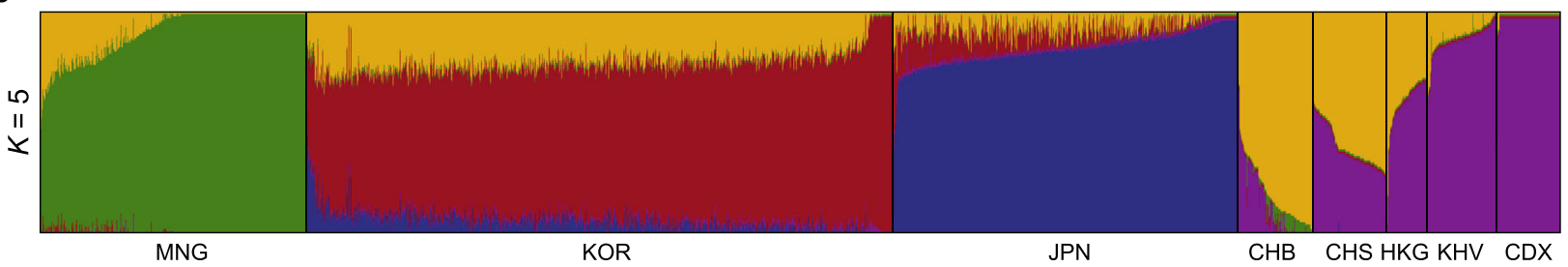

Fig. 1 Ancestry composition of 1779 individuals in the NARD. a PCA of global populations from the NARD and 1KGP3. AFR, AMR, EAS, EUR, and SAS denote Africans, Americans, East Asians, Europeans, and South Asians, respectively. b PCA of Northeast and Southeast Asians from the NARD and 1KGP3. Japanese in Tokyo from the 1KGP3 were combined into JPN. CHN from the NARD were categorized into CHB and CHS. c Population substructure of Northeast and Southeast Asians with five ancestral components inferred by ADMIXTURE algorithm

and Zakhchin (OTH, $n=12)$. They were also genetically separated into BUR and KHA/OTH (Additional file 1: Figure S8). The results highlight that the NARD has the most diverse genetic compositions of Northeast Asian populations by adding the two ancestries, KOR and MNG, which have been underrepresented in public datasets such as the 1KGP3 panel.

\section{Evaluation of NARD imputation panel}

To illustrate the robustness of the NARD as an imputation reference panel, we built a pseudo-GWAS dataset using an independent cohort of 97 unrelated KOR individuals $[15,19,20]$ and simulated the genotype imputation analysis. It was generated from WGS data by masking the genotypes that were not included in the sites of Illumina Omni $2.5 \mathrm{M}$ array. The imputation was conducted by Minimac3 [21] on the pre-phased SNPs using five types of reference panels: (1) NARD $(n=1779),(2) 1 \mathrm{KGP} 3(n=$ 2504), (3) HRC r1.1 $(n=32,470)$, (4) NARD + 1KGP3 $(n=4200)$, and (5) NARD + 1KGP3 (re-phased, $n=4200)$. To measure the imputation accuracy, we calculated the squared Pearson correlation coefficients $\left(R^{2}\right)$ between the true genotypes and the imputed dosages as a function of MAF in $850 \mathrm{KOR}$ individuals from the NARD. The imputation performance of the NARD exceeded the 1KGP3 panel for every MAF bin (Fig. 2a). Notably, the HRC panel, with the largest sample size including individuals from the 1KGP3, showed poor performance compared with other panels. Since the low imputation accuracy of the HRC panel is inconsistent with the original investigation, we performed the same analysis using 24 unrelated French (FRA) individuals [22]. In contrast to a KOR cohort, we confirmed that the HRC panel produced the most accurate genotype dosages for an FRA cohort, and the NARD panel had poor suitability for Europeans (Additional file 1: Figure S9).

We then merged the NARD and 1KGP3 panels and performed re-phasing to enhance the imputation performance based on the previous studies $[2,5]$. To combine the NARD and 1KGP3 panels without missing genotypes, we used the identical approach that was implemented in the UK10K and IMPUTE2 [5, 23]; we reciprocally imputed two panels using Minimac3 in order to statistically infer the missing genotypes in the NARD 


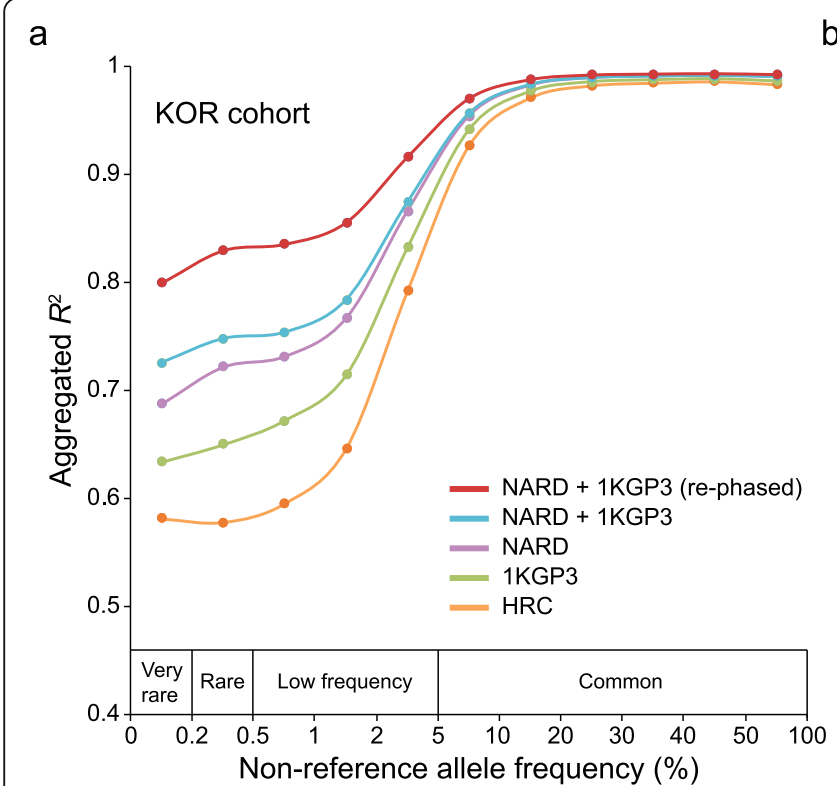

b

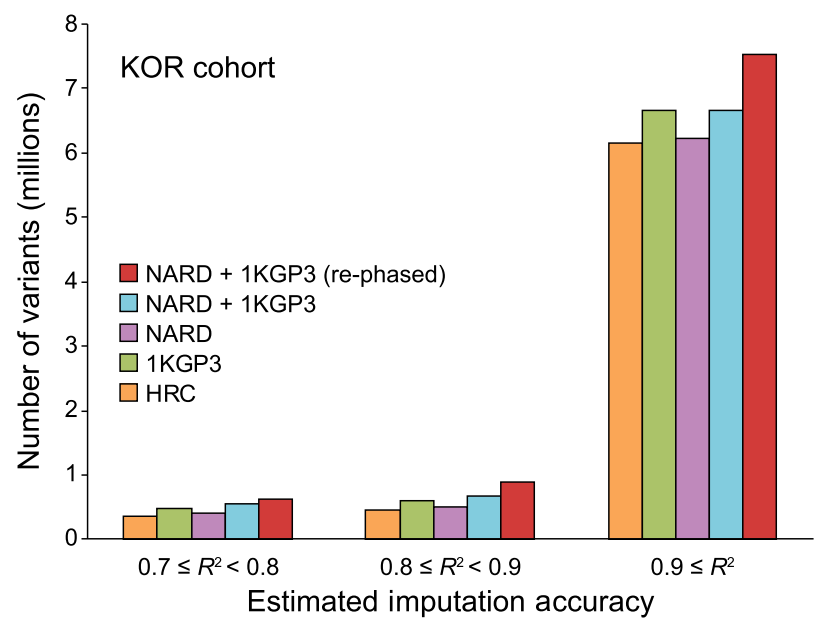

Fig. 2 Imputation performance evaluation. a Imputation accuracy assessment using the five different reference panels. The pseudo-GWAS panel of 97 KOR was used for the imputation. The $x$-axis represents MAF of 850 KOR individuals from the NARD. The $y$-axis represents the aggregated $R^{2}$ values of SNPS, which were calculated by the true genotypes and the imputed dosages. Only SNPs that were imputed across all panels were used for the aggregation of $R^{2}$ values. $\mathbf{b}$ Number of imputed SNPs as a function of the estimated imputation accuracy and the types of imputation panel. This result was generated based on the $R^{2}$ values that were estimated by Minimac3

or 1 KGP3 panels. Consistent with previous studies [3, 6, $8-10]$, combining the two panels showed more accurate imputation results compared with the NARD or $1 \mathrm{KGP3}$ alone. Furthermore, we confirmed a large improvement of the imputation accuracy, particularly for very rare $\left(\mathrm{MAF}<0.2 \% ; R^{2}=0.80\right)$, rare $\left(0.2 \% \leq \mathrm{MAF}<0.5 \% ; R^{2}=\right.$ $0.83)$, and low-frequency $\left(0.5 \% \leq \mathrm{MAF}<5 \% ; R^{2}=0.87\right)$ variants, when the haplotypes in the combined panel were re-phased by SHAPEIT3 [24]. In addition to measuring accuracy, we assessed the number of accurately imputed SNPs for each panel. For this analysis, we used the estimated $R^{2}$ values measured by Minimac3, as it is the standard for the quality control procedure in GWAS $[25,26]$. We found that the NARD + 1KGP3 (re-phased) panel produced the greatest number of high-confident SNPs $\left(R^{2} \geq 0.9\right)$ compared with other panels, especially $1 \mathrm{KGP} 3$ ( $n=7.5$ million versus 6.7 million), in concordance with the imputation accuracy (Fig. 2b).

We also illustrated the potential of the NARD + 1KGP3 (re-phased) as a reference panel for diverse Northeast Asians by performing additional imputation tests using independent cohorts of unrelated $\mathrm{CHN}$ and JPN individuals ( $n=79$ and 27, respectively) [27, 28]. For imputation accuracy measurement, we used MAF bins defined by 10,639 CHN and 3554 JPN individuals, respectively $[13,14]$. In agreement with the imputation result of a KOR cohort, the NARD + 1KGP3 (re-phased) panel provided the most accurate genotype imputation on very rare $\left(R^{2}=0.71\right.$ and 0.84 for $\mathrm{CHN}$ and JPN cohorts, respectively), rare $\left(R^{2}=0.71\right.$ and 0.89 for $\mathrm{CHN}$ and JPN cohorts, respectively), and low-frequency $\left(R^{2}=\right.$ 0.81 and 0.91 for CHN and JPN cohorts, respectively) variants (Additional file 1: Figure S10a and S10b). The NARD + 1KGP3 (re-phased) panel also generated the largest number of accurately imputed genotypes compared with other panels, particularly $1 \mathrm{KGP} 3(n=7.0 \mathrm{mil}-$ lion versus 6.8 million and 6.6 million versus 6.2 million for $\mathrm{CHN}$ and JPN cohorts, respectively; Additional file 1: Figure S10c and S10d).

To investigate where the improvement of the NARD + 1KGP3 (re-phased) comes from, we divided the panel into the NARD (re-phased) and 1KGP3 (re-phased) and assessed the imputation accuracy separately. The NARD (re-phased) panel had slightly lower imputation power than the NARD + 1KGP3 (re-phased) panel, but greatly improved compared to the original NARD panel (Additional file 2: Table S4). Meanwhile, the 1KGP3 (rephased) panel showed no improvement in the imputation accuracy compared to the original 1KGP3 panel.

We examined the underlying reasons for improved imputation performance caused by the re-phasing approach using identity-by-descent (IBD) analysis. It is known that phasing or genotype errors cause the gaps within the real IBD tracts; hence, the length of segments in phased genotype data tends to be shorter [29,30]. Based on this aspect, we expected that haplotype correction is occurred by re-phasing, and it would extend the length of shared IBD segments among individuals. Therefore, we 
measured the shared large IBD segments $(\geq 2 \mathrm{cM})$ between two individuals using the original (phased without 1KGP3) and re-phased haplotypes of the NARD. As a result, we confirmed the significant increase in length and number of shared IBD segments in re-phased haplotypes, which implies that the haplotype refinement in the NARD was achieved by the re-phasing process (Additional file 1: Figure S11).

\section{NARD imputation server}

We developed a user-friendly web site to provide imputation service using the NARD + 1KGP3 (re-phased) panel for researchers (Additional file 1: Figure S12). Our web site provides the imputation process for a wide range of genotype data format including PLINK (ped and bed files paired with map and bim/fam files, respectively) [31], 23andMe (Mountain View, CA), AncestryDNA (Lehi, UT), and variant call format (VCF) [32]. Results are processed through the imputation pipeline consisting of four major steps: pre-processing, phasing, imputation, and post-processing. The pre-processing step checks the format and content validity of uploaded files and converts them into VCF files for the next steps. Depending on the format of uploaded files, PLINK and 23andMe/AncestryDNA files will be converted into VCF files using GotCloud [33] and BCFtools [34], respectively, based on hg19 reference coordinate. When the input files have multiple chromosomes, the server will automatically separate them into multiple files. The subsequent analyses proceed regardless of whether files have "chr" prefix in their contig names or not. The preprocessed data is phased using Eagle2 [35] or SHAPEIT2 [36], and Beagle5.0 [37] with or without a reference panel, respectively. Then, imputation is performed with Minimac4 (https://github.com/statgen/Minimac4). In the post-processing step, the output is assessed and provided as bgzip-compressed VCF and PLINK binary files. The server will provide the PLINK format with extra files containing predicted $R^{2}$ values per variant for imputation quality check. Once imputation is finished, users will be notified by email and the result will be stored in the server for a week.

\section{NARD for variant interpretation}

Filtering common variants based on the population allele frequency is the first step to identify rare disease-causing genes [38]. To examine the potential advantage of NARD for clinical variant interpretation, the frequencies of SNPs between the Genome Aggregation Database (gnomAD, 2.1.1 release) [39] and NARD were compared. We redefined the frequency of 1.8 million genome-wide SNPs that are rare in worldwide populations from the gnomAD (gnomAD-ALL) to low-frequency or common $(\mathrm{MAF} \geq 5 \%)$. Moreover, 0.5 million rare genome-wide
SNPs in East Asians from the gnomAD (gnomAD-EAS) were low-frequency or common variants in the NARD (Fig. 3a). We simulated rare disease variant discovery using 203 samples that were included in the three pseudo-GWAS panels for the imputation analysis. We applied variant filtering criteria $(\mathrm{MAF}<5 \%)$ from the guidelines of the American College of Medical Genetics for the interpretation of sequence variants [40]. Notably, the number of protein-altering variants (missense, nonsense, frameshift, and splicing variants) was significantly reduced when the exome catalogues of gnomAD-EAS and NARD were jointly applied for variant filtration (Fig. 3b). This result represents that NARD could also contribute to the classification of pathogenic variant besides genotype imputation for the Northeast Asians.

\section{Whole-genome sequencing}

For 1690 individuals of KOR, JPN, MNG, and HKG, we performed WGS using Hiseq X instrument (Illumina, San Diego, CA) based on the manufacturer's instructions. We also used publicly available $91 \mathrm{CHN}$ samples [41], which were sequenced by Illumina Hiseq 2000 instrument (Illumina, San Diego, CA). This cohort consists of YH cell line and samples from the HapMap and 1KGP3 with high sequencing depth (on average, $70 \times$ ) $[1,42,43]$.

\section{Variant discovery and refinement}

Read alignment to the human reference genome (hg19) without any alternate contig, duplicate read removal, and joint calling of SNPs and indels were performed using Dynamic Read Analysis for GENomics platform (version 01.003.024.02.00.01.23004; http://edicogenome. $\mathrm{com} /$ dragen-bioit-platform/) with the following parameters: (i) creating gVCF: "--enable-map-align-output true," "--remove-duplicates true," "--enable-bam-indexing true, " "--enable-variant-caller true," and "--vc-emit-ref-confidence GVCF," and (ii) joint calling: "--enable-joint-genotyping true." For indels, we discarded variants greater than 49 base pairs, which are generally defined as structural variants [20,44]. Variant quality score recalibration (VQSR) was applied to raw variants based on the GATK's best practice [45] with the parameters given below:

i) Annotations

SNP: DP, QD, MQ, MQRankSum, FS, SOR

Indel: DP, QD, MQ, MQRankSum, ReadPosRankSum, FS, SOR

ii) Truth set SNP: HapMap3.3 and 1KGP Omni2.5

Indel: Mills \& 1KGP gold standard

iii) Training set SNP: HapMap3.3, 1KGP Omni2.5 and 1KGP phase1 high confidence 


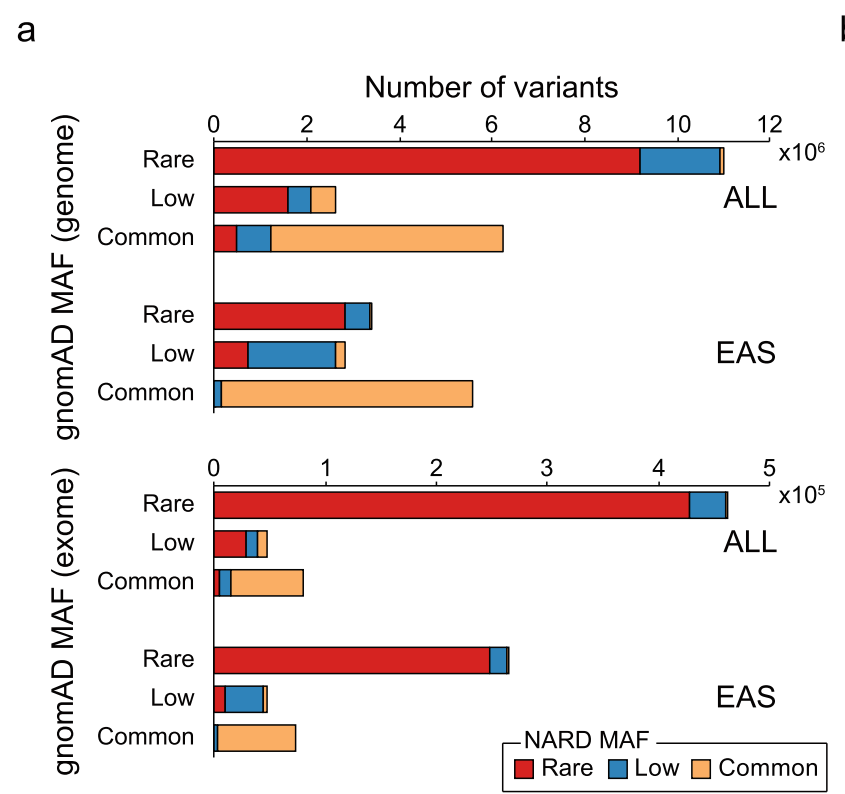

\section{b}

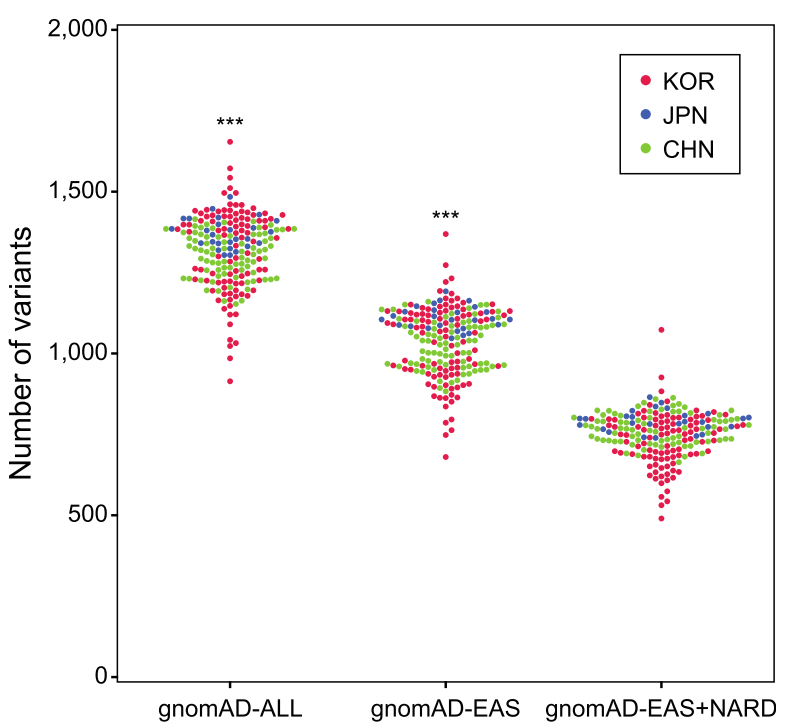

Fig. 3 Variant interpretation using the NARD. a MAF differences of SNPs shared between the NARD and gnomAD. The $y$-axis denotes the MAF of SNPs in worldwide populations (ALL) or EAS from the gnomAD. Color represents the MAF of SNPs in 1779 Northeast Asians from the NARD. $\mathbf{b}$ Number of uncommon (MAF < 5\%) protein-altering variants (missense, nonsense, frameshift, and splicing variants) after filtration using the gnomAD with/without NARD. Variant catalogue from the gnomAD (exome) was applied. ${ }^{* *} P<0.0001$ by two-tailed Mann-Whitney $U$ test (compared with gnomAD-EAS + NARD)

Indel: Mills \& 1 KGP gold standard and 1KGP phase 1

iv) Known set

SNP: dbsnp138

SNPs and indels below 99\% of truth sensitivity level from VQSR were initially filtered. Moreover, recalibrated variants were further filtered based on the following criteria: (i) located in the low complexity regions (LCRs), which were defined by the 1KGP3 study, (ii) genotype quality $<20$, and (iii) read depth $<5$. After these filtration processes, SNPs and indels were phased by SHAPEIT3 (version r884.1) which provides a fast population-scale phasing with low switch-error using the following model parameters: “--states 100," "--window 2," and "--effective-size 15000.”

\section{Variant discovery evaluation}

We selected the $86 \mathrm{CHN}$ samples in NARD with publicly available Illumina Omni $2.5 \mathrm{M}$ array data from the $1 \mathrm{KGP3}$, for validating variants in the NARD. A total of $1,664,330$ SNPs were overlapped between NARD and Omni chip, excluding mitochondrial DNA and pseudoautosomal regions. The concordance is the cumulative sum of the matching alleles divided by the total number of loci multiplied by two, which is the maximum matching opportunity in diploid. The sex chromosomes in male are considered as diploids for this calculation.

\section{Variant annotation}

All the SNPs and indels in this study were annotated by ANNOVAR based on the RefSeq gene definition [46, 47]. We annotated Kaviar [48], gnomAD, and The Single Nucleotide Polymorphism Database build 150 [27] for the classification of novel variants. For loss-of-function variant annotation, we implemented the Loss-Of-Function Transcript Effect Estimator (version 0.3-beta) [39] which is a plugin of Variant Effect Predictor [49] to remove low confidence annotations [50] with the following parameters: "--pick," “--vcf," "--cache," "--offline," and "--plugin LoF." For the 1KGP3 dataset, we also removed the variants within LCRs.

\section{Hardy-Weinberg equilibrium calculation}

We calculated HWE of variants in the NARD using VCFtools (version 0.1.12b) with "--hardy" option [32].

\section{Population structure analyses}

We converted VCF files of bi-allelic autosomal SNPs from the NARD and 1KGP3 into PLINK format using GotCloud (version 1.75.5). Then, we merged the two panels by PLINK (version 1.9) and extracted SNPs with genotype rate equals to $100 \%$ and $\mathrm{MAF} \geq 1 \%$ to remove the batch effect between the NARD and 1KGP3. Finally, we pruned SNPs with linkage disequilibrium $\left(R^{2}>0.1\right)$ within 50 base pairs sliding window using PLINK. 
With this processed data, we carried out PCA with Genome-wide Complex Trait Analysis (version 1.91.3beta) [51] using (1) worldwide populations from the NARD and 1KGP3 and (2) Northeast and Southeast Asians from the NARD and 1KGP3, separately. We also applied the unsupervised ADMIXTURE algorithm (version 1.3) for ancestry estimation. The optimal cluster number was determined by comparing the cross-validation error rates of each $K$ (Additional file 1: Figure S13). The results were visualized by Genesis (http://www.bioinf.wits.ac.za/software/genesis/).

\section{Imputation}

For the imputation panel, the singleton variants in the NARD were excluded, because they are difficult to be imputed. To combine the NARD and 1KGP3 panels, we used the same approach as the UK10K and IMPUTE2; NARD-specific variants were imputed into the 1KGP3 using Minimac3 (version 2.0.1) and vice versa, then they were merged into a single reference panel. In addition, the combined panel was re-phased by SHAPEIT3 using the model parameters mentioned above with "--earlystopping" and "--cluster-size 4000" parameters. We kept variants that are not located in LCRs.

We separately processed $113 \mathrm{KOR}, 79 \mathrm{CHN}, 27$ JPN, and 24. FRA individuals that are not included in the reference panels for imputation accuracy evaluation (Additional file 2: Table S3). Then, we discarded 16 related individuals from a KOR cohort. Unrelated sample selection was achieved by kinship estimation using KING [52]. Then, we extracted SNPs from sites on the Illumina Omni $2.5 \mathrm{M}$ array and monomorphic sites were excluded. As a result, 1,345,511, 1,320,123, 1,214,151, and $2,847,580$ autosomal SNPs remained in the pseudoGWAS panels of KOR, CHN, JPN, and FRA cohorts, respectively.

We performed imputation using Minimac3 with the five different types of reference panels. Imputation using the HRC panel was performed at the Michigan Imputation Server (https://imputationserver.sph.umich.edu). Before imputation, the haplotypes of individuals in the four cohorts were estimated using Eagle2 (version 2.3.2). After imputation, we extracted 4,352,921, 5,427,462, 48,431,56, and 5,419,512 SNPs in the four cohorts, which were imputed by all reference panels, and none of them were present with missing genotype in the non-masked dataset. The squared Pearson correlation coefficients $\left(R^{2}\right)$ were calculated between the imputed dosages and true genotypes, and those values were aggregated into 11 MAF bins to measure the imputation accuracy.

\section{IBD analysis}

The shared IBD segments between two individuals were identified using RefinedIBD (version 12Jul18.a0b) with "length $=2.0$ " parameter [29]. To evaluate the effect of the re-phasing approach on haplotype correction, we performed this analysis using the original and re-phased haplotypes of the NARD which were phased without and with the 1KGP3 panel, separately. The short gaps and breaks $(>0.6 \mathrm{cM})$ between IBD segments were discarded using merge-ibd-segments utility program.

\section{Utility and discussion}

Due to the cost-reduction and technological advancements in WGS, several groups have been focused on building the population-specific reference panels, especially for underrepresented populations in the conventional panels such as 1KGP3 [3, 4, 6-10, 13]. However, the Northeast Asian-specific reference panel with deep sequencing coverage and large sample size has been barely constructed and most of them are publicly unavailable. In this study, we integrated whole-genome sequence variants of 1779 Northeast Asian individuals to construct a reference panel, NARD; to resolve the uncertainty of genotype imputation along with the preexisting panels; and to facilitate more comprehensive genetic analysis of Northeast Asians.

Genotype imputation accuracy is known to be affected by several factors, and one of the major determinants is the sample size of reference panel $[5,36]$. Until now, most genotype imputations of Northeast Asians relied on the panels with large sample size [46-49], although the ancestries between the study population and reference panel are not matched. These panels showed lower imputation power, compared to the well-matched population-specific panels even with smaller sample size $[3,6-10,53]$. Considering the importance of populationspecific reference panel, we generated a large-scale WGS dataset of KOR and MNG that were not included in the $1 \mathrm{KGP3}$ panel. We confirmed that KOR and MNG were genetically differentiated from other East Asian populations. Therefore, the major ancestries in Northeast Asia are finally covered as population-scale by the NARD. In addition to the two populations, JPN, CHN, and HKG were also sequenced to increase the imputation power by the sample size effect and to build NARD as a reference panel that can be applied to diverse Northeast Asian populations.

Recently, the HRC panel was constructed using the genotypes of more than 30,000 individuals, mostly composed of European descent from various cohorts such as the 1KGP3 study. It is the largest publicly available reference panel, but previous studies demonstrated the poor imputation performance of this panel for $\mathrm{CHN}$, admixed Africans, and Hispanic/Latino populations, even worse than the 1KGP3 panel $[54,55]$, and our analysis again supported this result. It is reasonably different from the original investigation of the HRC study because they only examined the imputation 
accuracy using European ancestry. The inconsistent results between the HRC study and others imply that several complex properties should be considered for achieving highquality genotype imputation. It could be speculated that the population specificity between the reference panel and the individuals to be imputed would be occasionally more relevant factor than the size of reference panel. Therefore, the HRC panel might not be a gold standard for non-European populations. We believe that our new reference panel and analysis are valuable resources for researchers who want to achieve more accurate genotype imputation in Northeast Asians.

As previous studies yield further increment of the imputation accuracy from their population-specific panels by combining dataset of the $1 \mathrm{KGP} 3[3,6,8-10]$, we also confirmed the improvement of the imputation performance by combining the NARD and 1KGP3 panels using a fast and simple approach as described in the UK10K and IMPUTE2. However, there could be an issue regarding the uncertainty of imputed genotypes, since the missing genotypes in each panel were statistically estimated. Referring to the HRC study, calculating genotype likelihood of each variant using the individual BAM files would improve the uncertainty of these genotypes, if the sequencing coverages are sufficient. After merging the NARD and 1KGP3, we enhanced the power of the combined panel by applying the re-phasing strategy. It is an advanced process that has not been applied in most of previous studies $[3,6,8-10]$, but the HRC study has shown further improvement of the imputation accuracy with this approach. Based on this strategy, the NARD + 1KGP3 (re-phased) panel produced more accurately imputed genotype dosages, especially for uncommon variants $(\mathrm{MAF}<5 \%)$, than the NARD + 1 KGP3 panel. This might be due to haplotype correction with the assistance of the haplotypes in the 1KGP3 panel.

\section{Conclusions}

In summary, we generated a large-scale reference panel for Northeast Asians, which will be a highly valuable resource to resolve a persistent deficiency of Asian genome data. We believe that our efforts will remarkably contribute to precision medicine in Northeast Asia.

\section{Supplementary information}

Supplementary information accompanies this paper at https://doi.org/10. 1186/s13073-019-0677-z.

Additional file 1: Figure S1. Geographic map of the study area in the NARD. Figure S2. Correlation between the sequencing depth and number of variants. Figure S3. Transition to transversion ratio of the populations in the NARD. Figure S4. Heterozygous to homozygous ratio of the global populations. Figure S5. Number of loss-of-function variants. Figure S6. Hardy-Weinberg Equilibrium test of variants in the NARD. Figure S7. Novel variant statistics. Figure S8. Differential genetic composition of the two MNG groups. Figure S9. Imputation performance evaluation of FRA individuals. Figure S10. Imputation performance evaluation of CHN and JPN individuals. Figure S11. Length distribution of shared IBD tracts between the two individuals in each population. Figure S12. The flow chart of the pipeline consisting of four major steps for NARD imputation server. Figure S13. The cross-validation error inferred by ADMIXTURE algorithm.

Additional file 2: Table S1. Variant statistics. Table S2. Variant accuracy. Table S3. Data source for imputation analysis. Table S4. Imputation performance according to types of reference panel.

\section{Abbreviations}

NARD: Northeast Asian Reference Database; GWAS: Genome-wide association study; 1KGP3: 1000 Genomes Project Phase 3; WGS: Whole-genome sequencing; HRC: Haplotype Reference Consortium; CHN: Han Chinese; JPN: Japanese; MNG: Mongolians; KOR: Koreans; HKG: Hong Kongese; Het/ Hom: Heterozygous to homozygous genotypes; SNP: Single nucleotide polymorphism; Indel: Short insertion/deletion; Ti/TV: Transition to transversion; HWE: Hardy-Weinberg equilibrium; MAF: Minor allele frequency; PCA: Principal component analysis; CHB: Han Chinese in Beijing; CHS: Han Chinese in Shanghai; KHV: Kinh in Ho Chi Minh City; BUR: Buryats; KHA: Khalkha Mongols; OTH: Other Mongolians; FRA: French; IBD: Identity-bydescent; VCF: Variant call format; gnomAD: Genome Aggregation Database; gnomAD-ALL: Worldwide populations from the gnomAD; gnomAD-EAS: East Asians from the gnomAD; VQSR: Variant quality score recalibration; LCR: Low complexity region

\section{Acknowledgements}

We thank the members of GenomAsia100K Consortium for the discussion and assistance with the manuscript preparation.

\section{Authors' contributions}

J-SS, CK, SS, J-IK, FM, and BC designed the project. S-KY, C-UK, HLK, and SK wrote the manuscript. S-KY, C-UK, HLK, and SK performed the bioinformatic analysis. C-UK and NK built the imputation server. J-YS performed the library preparation and next-generation sequencing. K-WL and JSY contributed to data interpretation. All authors read and approved the final manuscript.

\section{Funding}

F.M. is supported by the Japan Agency for Medical Research and Development under grant numbers JP18kk0205008h0003,

JP16ek0109070h0003, and JP18ek0109283h0001. K.-W.L. is funded by the Research Grant Council, Hong Kong (Theme-based Research Scheme: T12401/13-R).

\section{Availability of data and materials}

Raw sequence data is protected and cannot be released to the public due to compromise of participant confidentiality and privacy. Alternatively, genotype imputation can be freely performed at the NARD imputation server for the academic purpose (https://nard.macrogen.com/). Researchers can download MAF data from the NARD as a VCF file (https://nard. macrogen.com/download/NARD_MAF.hg19.zip). The hg38 version of MAF data liftovered by CrossMap (version 0.3.6) [56] is also available (https://nard. macrogen.com/download/NARD_MAF.hg38.zip).

\section{Ethics approval and consent to participate}

This study was approved by the institutional review board of Seoul National University Hospital, in accordance with the Declaration of Helsinki (approved ID: C-1705-048-852). Written informed consent was obtained from all study subjects.

\section{Consent for publication}

Not applicable.

\section{Competing interests}

The authors affiliated with Precision Medicine Institute are full-time employees at Macrogen: S.-K.Y., C.-U.K., S.K., J.-Y.S., N.K., J.S.Y., C.K., and J.-S.S. The remaining authors declare that they have no competing interests. 


\section{Author details}

${ }^{1}$ Precision Medicine Center, Seoul National University Bundang Hospital, 172 Dolma-ro, Seongnam, Bundang-gu, Gyeonggi-do 13605, Republic of Korea. ${ }^{2}$ Precision Medicine Institute, Macrogen Inc., Seongnam, Republic of Korea. ${ }^{3}$ Department of Biomedical Sciences, Seoul National University Graduate School, Seoul, Republic of Korea. ${ }^{4}$ The Asian School of the Environment, Nanyang Technological University, Singapore, Singapore. ${ }^{5}$ Singapore Centre for Environmental Life Sciences Engineering, Nanyang Technological University, Singapore, Singapore. ${ }^{6}$ Department of Anatomical \& Cellular Pathology and State Key Laboratory of Translational Oncology, The Chinese University of Hong Kong, Hong Kong, China. ${ }^{7}$ Department of Family Medicine, Seoul National University Hospital, Seoul, Republic of Korea. ${ }^{8}$ Center for Genomic Medicine, Kyoto University Graduate School of Medicine, Kyoto, Japan. ${ }^{9}$ School of Biological Science, Nanyang Technological University, Singapore, Singapore. ${ }^{10} \mathrm{Genomic}$ Medicine Institute, Medical Research Center, Seoul National University, Seoul, Republic of Korea. ${ }^{11}$ Gong-Wu Genomic Medicine Institute, Seoul National University Bundang Hospital, Seongnam, Republic of Korea.

Received: 8 May 2019 Accepted: 11 October 2019

Published online: 22 October 2019

\section{References}

1. The 1000 Genomes Project Consortium. A global reference for human genetic variation. Nature. 2015;526:68-74.

2. McCarthy S, Das S, Kretzschmar W, Delaneau O, Wood AR, Teumer A, Kang HM, Fuchsberger C, Danecek P, Sharp K, et al. A reference panel of 64,976 haplotypes for genotype imputation. Nat Genet. 2016;48: $1279-83$.

3. The Genome of the Netherlands Consortium. Whole-genome sequence variation, population structure and demographic history of the Dutch population. Nat Genet. 2014;46:818-25.

4. Gudbjartsson DF, Helgason H, Gudjonsson SA, Zink F, Oddson A, Gylfason A, Besenbacher S, Magnusson G, Halldorsson BV, Hjartarson E, et al. Large-scale whole-genome sequencing of the Icelandic population. Nat Genet. 2015;47: 435-44.

5. Huang J, Howie B, McCarthy S, Memari Y, Walter K, Min JL, Danecek P, Malerba G, Trabetti E, Zheng HF, et al. Improved imputation of lowfrequency and rare variants using the UK10K haplotype reference panel. Nat Commun. 2015;6:8111.

6. Nagasaki M, Yasuda J, Katsuoka F, Nariai N, Kojima K, Kawai Y, YamaguchiKabata Y, Yokozawa J, Danjoh I, Saito S, et al. Rare variant discovery by deep whole-genome sequencing of 1,070 Japanese individuals. Nat Commun. 2015;6:8018.

7. Sidore C, Busonero F, Maschio A, Porcu E, Naitza S, Zoledziewska M, Mulas A, Pistis G, Steri M, Danjou F, et al. Genome sequencing elucidates Sardinian genetic architecture and augments association analyses for lipid and blood inflammatory markers. Nat Genet. 2015;47:1272-81.

8. Hou L, Kember RL, Roach JC, O'Connell JR, Craig DW, Bucan M, Scott WK, Pericak-Vance $\mathrm{M}$, Haines $\mathrm{J}$, Crawford $\mathrm{MH}$, et al. A population-specific reference panel empowers genetic studies of Anabaptist populations. Sci Rep. 2017;7:6079.

9. Mitt M, Kals M, Parn K, Gabriel SB, Lander ES, Palotie A, Ripatti S, Morris AP, Metspalu A, Esko T, et al. Improved imputation accuracy of rare and lowfrequency variants using population-specific high-coverage WGS-based imputation reference panel. Eur J Hum Genet. 2017;25:869-76.

10. Bai H, Guo X, Narisu N, Lan T, Wu Q, Xing Y, Zhang Y, Bond SR, Pei Z, Zhang $Y$, et al. Whole-genome sequencing of 175 Mongolians uncovers population-specific genetic architecture and gene flow throughout North and East Asia. Nat Genet. 2018;50:1696-704.

11. Sirugo $G$, Williams $S M$, Tishkoff SA. The missing diversity in human genetic studies. Cell. 2019;177:26-31.

12. Liu S, Huang S, Chen F, Zhao L, Yuan Y, Francis SS, Fang L, Li Z, Lin L, Liu R, et al. Genomic analyses from non-invasive prenatal testing reveal genetic associations, patterns of viral infections, and Chinese population history. Cell. 2018;175:347-59 e314.

13. Okada Y, Momozawa Y, Sakaue S, Kanai M, Ishigaki K, Akiyama M, Kishikawa T, Arai Y, Sasaki T, Kosaki K, et al. Deep whole-genome sequencing reveals recent selection signatures linked to evolution and disease risk of Japanese. Nat Commun. 2018;9:1631.
14. Cai N, Bigdeli TB, Kretzschmar WW, Li Y, Liang J, Hu J, Peterson RE, Bacanu S, Webb BT, Riley B, et al. 11,670 whole-genome sequences representative of the Han Chinese population from the CONVERGE project. Sci Data. 2017:4:170011.

15. Kim J, Weber JA, Jho S, Jang J, Jun J, Cho YS, Kim HM, Kim H, Kim Y, Chung O, et al. KoVariome: Korean National Standard Reference Variome database of whole genomes with comprehensive SNV, indel, CNV, and SV analyses. Sci Rep. 2018;8:5677

16. Lee S, Seo J, Park J, Nam JY, Choi A, Ignatius JS, Bjornson RD, Chae JH, Jang IJ, Lee $S$, et al. Korean Variant Archive (KOVA): a reference database of genetic variations in the Korean population. Sci Rep. 2017;7:4287.

17. Kwak SH, Chae J, Choi S, Kim MJ, Choi M, Chae JH, Cho EH, Hwang TJ, Jang SS, Kim Jl, et al. Findings of a 1303 Korean whole-exome sequencing study. Exp Mol Med. 2017;49:e356.

18. Alexander $\mathrm{DH}$, Novembre J, Lange K. Fast model-based estimation of ancestry in unrelated individuals. Genome Res. 2009;19:1655-64.

19. Ju YS, Kim Jl, Kim S, Hong D, Park H, Shin JY, Lee S, Lee WC, Kim S, Yu SB, et al. Extensive genomic and transcriptional diversity identified through massively parallel DNA and RNA sequencing of eighteen Korean individuals. Nat Genet. 2011;43:745-52.

20. Seo JS, Rhie A, Kim J, Lee S, Sohn MH, Kim CU, Hastie A, Cao H, Yun JY, Kim J, et al. De novo assembly and phasing of a Korean human genome. Nature. 2016;538:243-7.

21. Das S, Forer L, Schonherr S, Sidore C, Locke AE, Kwong A, Vrieze SI, Chew EY, Levy S, McGue M, et al. Next-generation genotype imputation service and methods. Nat Genet. 2016;48:1284-7.

22. Cann HM, de Toma C, Cazes L, Legrand MF, Morel V, Piouffre L, Bodmer J, Bodmer WF, Bonne-Tamir B, Cambon-Thomsen A, et al. A human genome diversity cell line panel. Science. 2002;296:261-2.

23. Howie BN, Donnelly P, Marchini J. A flexible and accurate genotype imputation method for the next generation of genome-wide association studies. PLoS Genet. 2009;5:e1000529.

24. O'Connell J, Sharp K, Shrine N, Wain L, Hall I, Tobin M, Zagury JF, Delaneau O, Marchini J. Haplotype estimation for biobank-scale data sets. Nat Genet. 2016;48:817-20.

25. CONVERGE consortium. Sparse whole-genome sequencing identifies two loci for major depressive disorder. Nature. 2015;523:588-91.

26. Son HY, Hwangbo Y, Yoo SK, Im SW, Yang SD, Kwak SJ, Park MS, Kwak SH, Cho SW, Ryu JS, et al. Genome-wide association and expression quantitative trait loci studies identify multiple susceptibility loci for thyroid cancer. Nat Commun. 2017:8:15966.

27. Sherry ST, Ward MH, Kholodov M, Baker J, Phan L, Smigielski EM, Sirotkin K. dbSNP: the NCBI database of genetic variation. Nucleic Acids Res. 2001;29: 308-11.

28. Lu D, Lou H, Yuan K, Wang X, Wang Y, Zhang C, Lu Y, Yang X, Deng L, Zhou $Y$, et al. Ancestral origins and genetic history of Tibetan highlanders. Am J Hum Genet. 2016;99:580-94.

29. Bjelland DW, Lingala U, Patel PS, Jones M, Keller MC. A fast and accurate method for detection of IBD shared haplotypes in genome-wide SNP data. Eur J Hum Genet. 2017;25:617-24.

30. Browning BL, Browning SR. Improving the accuracy and efficiency of identity-by-descent detection in population data. Genetics. 2013;194:459-71.

31. Chang CC, Chow CC, Tellier LC, Vattikuti S, Purcell SM, Lee JJ. Secondgeneration PLINK: rising to the challenge of larger and richer datasets. Gigascience. 2015;4:7.

32. Danecek P, Auton A, Abecasis G, Albers CA, Banks E, DePristo MA, Handsaker RE, Lunter G, Marth GT, Sherry ST, et al. The variant call format and VCFtools. Bioinformatics. 2011;27:2156-8.

33. Jun G, Wing MK, Abecasis GR, Kang HM. An efficient and scalable analysis framework for variant extraction and refinement from population-scale DNA sequence data. Genome Res. 2015;25:918-25.

34. Li H, Handsaker B, Wysoker A, Fennell T, Ruan J, Homer N, Marth G, Abecasis G, Durbin R. Genome project data processing S: the sequence alignment/ map format and SAMtools. Bioinformatics. 2009;25:2078-9.

35. Loh PR, Danecek P, Palamara PF, Fuchsberger C, Reshef YA, Finucane HK, Schoenherr S, Forer L, McCarthy S, Abecasis GR, et al. Reference-based phasing using the Haplotype Reference Consortium panel. Nat Genet. 2016; 48:1443-8.

36. Delaneau O, Zagury JF, Marchini J. Improved whole-chromosome phasing for disease and population genetic studies. Nat Methods. 2013;10:5-6.

37. Browning $B L$, Zhou Y, Browning SR. A one-penny imputed genome from next-generation reference panels. Am J Hum Genet. 2018;103:338-48. 
38. Boycott KM, Vanstone MR, Bulman DE, MacKenzie AE. Rare-disease genetics in the era of next-generation sequencing: discovery to translation. Nat Rev Genet. 2013;14:681-91.

39. Karczewski KJ, Francioli LC, Tiao G, Cummings BB, Alföldi J, Wang Q, Collins RL, Laricchia KM, Ganna A, Birnbaum DP, et al. Variation across 141,456 human exomes and genomes reveals the spectrum of loss-of-function intolerance across human protein-coding genes. bioRxiv. 2019:531210. https://www.biorxiv.org/content/10.1101/531210v2.

40. Richards S, Aziz N, Bale S, Bick D, Das S, Gastier-Foster J, Grody WW, Hegde $M$, Lyon E, Spector $E$, et al. Standards and guidelines for the interpretation of sequence variants: a joint consensus recommendation of the American College of Medical Genetics and Genomics and the Association for Molecular Pathology. Genet Med. 2015;17:405-24.

41. Lan T, Lin H, Zhu W, Laurent T, Yang M, Liu X, Wang J, Wang J, Yang H, Xu $X$, Guo X. Deep whole-genome sequencing of $90 \mathrm{Han}$ Chinese genomes. Gigascience. 2017;6:1-7.

42. The International HapMap Consortium. A haplotype map of the human genome. Nature. 2005;437:1299-320.

43. Wang J, Wang W, Li R, Li Y, Tian G, Goodman L, Fan W, Zhang J, Li J, Zhang J, et al. The diploid genome sequence of an Asian individual. Nature. 2008;456:60-5.

44. The 1000 Genomes Project Consortium. A map of human genome variation from population-scale sequencing. Nature. 2010;467:1061-73.

45. DePristo MA, Banks E, Poplin R, Garimella KV, Maguire JR, Hartl C, Philippakis AA, del Angel G, Rivas MA, Hanna M, et al. A framework for variation discovery and genotyping using next-generation DNA sequencing data. Nat Genet. 2011:43:491-8.

46. Wang $\mathrm{K}, \mathrm{Li} \mathrm{M}$, Hakonarson $\mathrm{H}$. ANNOVAR: functional annotation of genetic variants from high-throughput sequencing data. Nucleic Acids Res. 2010;38:e164.

47. Bray RA, Hurley CK, Kamani NR, Woolfrey A, Muller C, Spellman S, Setterholm M, Confer DL. National marrow donor program HLA matching guidelines for unrelated adult donor hematopoietic cell transplants. Biol Blood Marrow Transplant. 2008; 14:45-53.

48. Glusman G, Caballero J, Mauldin DE, Hood L, Roach JC. Kaviar: an accessible system for testing SNV novelty. Bioinformatics. 2011;27:3216-7.

49. McLaren W, Gil L, Hunt SE, Riat HS, Ritchie GR, Thormann A, Flicek P, Cunningham F. The Ensembl variant effect predictor. Genome Biol. 2016;17:122.

50. MacArthur DG, Balasubramanian S, Frankish A, Huang N, Morris J, Walter K, Jostins L, Habegger L, Pickrell JK, Montgomery SB, et al. A systematic survey of loss-of-function variants in human protein-coding genes. Science. 2012;335:823-8.

51. Yang J, Lee SH, Goddard ME, Visscher PM. GCTA: a tool for genome-wide complex trait analysis. Am J Hum Genet. 2011;88:76-82.

52. Manichaikul A, Mychaleckyj JC, Rich SS, Daly K, Sale M, Chen WM. Robust relationship inference in genome-wide association studies. Bioinformatics. 2010;26:2867-73

53. Yasuda J, Katsuoka F, Danjoh I, Kawai Y, Kojima K, Nagasaki M, Saito S, Yamaguchi-Kabata Y, Tadaka S, Motoike IN, et al. Regional genetic differences among Japanese populations and performance of genotype imputation using whole-genome reference panel of the Tohoku Medical Megabank Project. BMC Genomics. 2018;19:551.

54. Kowalski MH, Qian H, Hou Z, Rosen JD, Tapia AL, Shan Y, Jain D, Argos M, Arnett DK, Avery C, et al. Use of $>100,000$ NHLBI Trans-Omics for Precision Medicine (TOPMed) Consortium whole genome sequences improves imputation quality and detection of rare variant associations in admixed African and Hispanic/Latino populations. bioRxiv. 2019:683201. https:/www. biorxiv.org/content/10.1101/683201v1

55. Lin Y, Liu L, Yang S, Li Y, Lin D, Zhang X, Yin X. Genotype imputation for Han Chinese population using Haplotype Reference Consortium as reference. Hum Genet. 2018;137:431-6.

56. Zhao H, Sun Z, Wang J, Huang H, Kocher JP, Wang L. CrossMap: a versatile tool for coordinate conversion between genome assemblies. Bioinformatics. 2014;30:1006-7.

\section{Publisher's Note}

Springer Nature remains neutral with regard to jurisdictional claims in published maps and institutional affiliations. 